\title{
PRODUCCIÓN INFORMATIVA EN SALUD: PERIODISMO RADIOFÓNICO EN PUEBLA
}

\author{
Health Information Production: Radio Journalism in Puebla \\ Produção informativa em saúde: periodismo radiofónico em Puebla
}

ROMERO CÁRCAMO, Lucano. Universidad Iberoamericana. (México)

superstarlion@gmail.com

Fecha de recibido: 30 de julio de 2015

Fecha de aceptado: 06 de octubre de 2015

\section{RESUMEN}

El estudio explora las salas de redacción y cabinas de locución en 11 radiodifusoras de las tres principales ciudades del Estado de Puebla, que son: Puebla, Tehuacán y Teziutlán, en cuyos programas se difunden contenidos relacionados con salud. Se integró un análisis cualitativo basado en: a) observación participante y b) entrevistas en profundidad a 20 periodistas y comunicadores en conjunto. El proceso de producción informativa fue investigado con base en las rutinas de producción, donde se indagó la operatividad y cultura de convergencia de las salas de redacción de cada radiodifusora: cómo se genera la información en salud y cómo se jerarquiza. Además, se analizó el papel de la política organizacional vinculada a la línea editorial.

Al investigar el fenómeno, se encontró una notable dependencia a las fuentes institucionales del sector médico; además, la información en salud no es considerada prioritaria debido a la poca relevancia editorial que se le otorga, así como a la falta de especialización de periodistas y comunicadores. Investigaciones sobre la cobertura periodística y divulgación en salud apuntan a que privilegiar ciertas fuentes informativas perjudica el profesionalismo de los periodistas.

Palabras clave: Puebla, salud, medios, producción, radio. 


\section{ABSTRACT}

The study explores the newsrooms and voiceover booths in 11 radio stations in the three major cities of Puebla, which are: Puebla, Tehuacan and Teziutlán, which broadcast health-related content. It is a qualitative analysis grounded on: a) participant observation, and b) in-depth interviews to 20 journalists and communicators together. The process of news production was investigated based on its production routines, where the operation and culture of convergence of each broadcaster's newsroom was investigated: how health information is generated and how it is nested. The role of organizational policy linked to the editorial line was also analyzed.

Researching about this phenomenon, a remarkable dependence on institutional sources in the medical institutions was found. Also, health information is not considered a priority due to the lack of relevance publishers give to it, as well as for the lack of specialization of journalists and communicators. Media coverage research and outreach in health suggest that favoring certain media sources causes serious damage to the professionalism of journalists.

Keywords: Puebla, health, media, production, radio.

\section{RESUMO}

O estudo explora as salas de redação e cabinas de locução em 11 radiodifusoras das três principais cidades do Estado de Puebla, que são: Puebla, Tehuacán e Teziutlán, em cujos programas se difundem conteúdos relacionados com saúde. Integrou-se uma análise qualitativa baseada em: a) observação participante e b) entrevistas em profundidade a 20 jornalistas e comunicadores em conjunto. 0 processo de produção informativa foi investigado com base nas rotinas de produção, onde indagou-se a operatividade e cultura de convergência das salas de redação de cada radiodifusora: como se gera a informação em saúde e como se hierarquiza. Além disso, analisou-se o papel da política organizacional vinculada à linha editorial.

Ao investigar o fenómeno, encontrou-se uma notável dependência às fontes institucionais do setor médico; além disso a informação em saúde não é considerada prioritária devido à pouca relevância editorial que se lhe outorga, assim como à falta de especialização de jornalistas e comunicadores. Pesquisas sobre a cobertura jornalística e divulgação em saúde apontam a que privilegiar certas fontes informativas prejudica o profissionalismo dos jornalistas.

Palavras-chave: Puebla, saúde, meios, produção, rádio. 


\section{Introducción}

Los medios de comunicación ocupan, tras los profesionales médicos, el segundo lugar como fuente de información sanitaria (Orueta et al., 2011) debido a que abordan temas relativos a salud. Su importancia ha trascendido a tal punto, que son el punto de mira de las agencias de noticias, e incluso de los mismos médicos (Montes de Oca, 2004).

Estudios previos, con México como centro de interés, han advertido sobre el carácter burocrático y rutinario del trabajo cotidiano de los periodistas (Hughes, 2009) lo que contrasta con el ideal del periodista investigador y de campo (Salinas, 2008). Lo anterior incide en el grado de homogeneidad de las noticias, tanto a nivel formal como de contenido; lo cual apunta a la presencia de similitudes de los periodistas y comunicadores en cuanto a rutinas, criterios y procesos editoriales, así como a formas de socialización con las fuentes informativas. De ahí la necesidad de investigar sobre las formas de producción que tienen periodistas y comunicadores en salud, pues la disponibilidad de profesionales especializados en esa área garantizará la presencia continuada de esta información con la generación de historias de mayor interés, profundidad y diversidad.

La presente investigación tiene el objetivo principal de identificar cómo se desarrolla el proceso de producción de los temas en salud en las salas de redacción y cabinas de locución de 11 radiodifusoras en conjunto de las tres principales ciudades del Estado de Puebla, que de acuerdo con datos del Instituto Nacional de Estadística, Geografía e Informática (INEGI, 2010) son: Puebla (capital), Tehuacán y Teziutlán. ¿Cuáles son las prácticas y/o rutinas de producción que periodistas y comunicadores en salud realizan en las estaciones de radio en las que trabajan?, ¿qué función tienen la línea editorial y la política organizacional de las radiodifusoras en relación a cómo abordar los contenidos en salud? El origen de esta investigación plantea que los comunicadores en salud, que trabajan en radio, producen contenidos privilegiando el acceso a las fuentes institucionales públicas y a representantes del sector médico.

Para verificar el fenómeno, el primer apartado desarrolla las categorías teórico-conceptuales necesarias para hacer visible nuestro objeto de estudio, tales como la sociología de la producción de noticias y la cultura periodística. Esto resulta útil para comprender de mejor manera las rutinas de producción puestas en marcha en el periodismo en salud en el Estado de Puebla. En la segunda parte se revisa el contexto sociodemográfico en el que se encuentra la radio en dicho Estado y cómo ello determina el tipo de prácticas periodísticas y la manera en que asumen su labor quienes trabajan en la radio. El tercer apartado centra su interés en la metodología empleada en esta investigación. Con los métodos cualitativos de la observación participante y la entrevista en profundidad se acompañó a periodistas y comunicadores en su labor cotidiana de producir noticias y programas de salud. Se realizaron entrevistas a 20 periodistas y comunicadores de 11 estaciones de radio en conjunto de las ciudades de Puebla, Tehuacán y Teziutlán. La forma en que se aplicaron tales herramientas metodológicas es parte integral de esta sección.

La cuarta sección inicia con los hallazgos de la investigación: se explica el papel protagónico que están asumiendo las fuentes informativas oficiales en las prácticas periodísticas; se expone la cultura de convergencia que presentan las radiodifusoras investigadas (los aspectos en que resultan similares las rutinas de producción informativa en salud); se explora la necesidad de la especialización periodística, que implica retos en cuanto a que informar en salud refiere una tarea compleja. 
Al cierre de esta investigación se presentan la serie de conclusiones y recomendaciones que se consideran pertinentes, con miras a contribuir en el campo del periodismo especializado en salud dentro del contexto regional. El trabajo contribuye en el conocimiento de un trozo de esa realidad mediática identificando algunas de sus características, así como algunos aspectos fundamentales para su desarrollo.

\section{Aproximación teórica}

Según Hernández (1997), las primeras reflexiones sociológicas sobre la producción de las noticias se ubicaron en la década de los años setenta bajo el nombre de Media Sociology, cuyo propósito era dar cuenta de los factores sociales que intervienen e influyen en los procesos de producción de los contenidos informativos de los medios de comunicación. La producción de noticias, explica Schudson (2003), resulta de la constante interacción entre periodistas y otros actores institucionales involucrados: voceros de información pública, empresas de relaciones públicas y demás fuentes periodísticas. El proceso de producción continúa con la interacción que deviene con otros agentes: directivos, editores, audiencias y todo el conjunto de entidades que componen la sociedad y el contexto sobre el cual se informa. Es ahí donde la sociología ha contribuido en entender a las noticias, especialmente al concentrarse en cómo los periodistas y comunicadores construyen e interpretan la realidad.

Para el caso que nos interesa no se trata de pensar en los contenidos informativos radiofónicos en salud como inventados, pues las noticias son una realidad construida y cuyos componentes son los hechos; en otras palabras, información pertinente recolectada por métodos validados profesionalmente (Tuchman, 1978). De ahí que la sociología de la producción de las noticias sea el enfoque adecuado para el fenómeno aquí desarrollado. La diversidad de prácticas periodísticas también pueden ser analizadas a partir de la cultura periodística, esto en cuanto a la forma en la que periodistas y comunicadores trabajan.

Estudios previos han reconocido que las normas culturales influyen la manera en la que los periodistas perciben su labor (Weaver, 1998, citado en Herscovitz, 2004). Hanitzsch (2007) construye el concepto de cultura periodística, la cual se examina a partir de las prácticas de periodistas y comunicadores, que llegan a ser influenciadas por sus percepciones. Hanitzsch indica que "la cultura periodística es más que una ideología” (Hanitzsch, 2007, p. 370$)^{1}$, pues integra un conjunto de orientaciones, valores, cualidades y prácticas presentes en el trabajo de los profesionales de la información, que les permite formar su acción profesional.

Existen investigaciones que se han dado a la tarea de analizar la noción de cultura periodística. Waisbord (2000) identificó que el periodismo resulta más un periodismo vigilante que investigativo, éste último en términos de la tradición periodística anglosajona, pues las explicaciones de los reporteros son "resultado de una cultura periodística obsesionada con los tratos del gobierno" (Waisbord, p. 88)². Según el autor, el proceso, siempre complejo, de producción de las noticias sobre el mal actuar de las autoridades gubernamentales requiere analizar la recolección de información, particularmente la relación que existe entre las salas de redacción y sus fuentes. Para comprender las culturas de producción periodísticas es necesario concebir a los periodistas como productores

1 Traducción propia.

2 Traducción propia. 
culturales, capaces de construir y transmitir significados, a la vez que articulan y difunden las ideologías que identifican a una comunidad política y social de una nación.

Otras investigaciones han evidenciado que los medios de comunicación ejercen una función determinante en el actuar de las audiencias cuando estas deben tomar decisiones sobre el cuidado de su salud (Linden, 2003; Mercado Martínez et al., 2001; Alcalay \& Mendoza, 2000). Las audiencias expuestas a las noticias adquieren conocimientos sobre salud y pueden cambiar sus actitudes (Hornik, 1991).

La investigación sobre la práctica de la producción del periodismo especializado en salud en México poco se ha reflexionado de forma sistemática respecto a la formación profesional. Las pocas investigaciones en México sobre el periodismo en salud concluyen que las notas relacionadas con la divulgación de dicho tema carecen de una visión crítica, son insuficientes, parciales y confusas (González \& Hernández, 2013). Esto teniendo en cuenta que en México los medios de comunicación, en su mayoría, aluden a asuntos de interés político y económico (Mercado Martínez et al., 2001). Por eso resulta imprescindible analizar las formas en las que se producen los contenidos informativos en materia de salud, particularmente desde la radio. La naturaleza de la radio, así como su constitución, hacen ideal ese medio de comunicación para realizar la cobertura y difusión de los temas de salud, sobre todo en un país como México, donde existe una enorme tradición de escuchar radio (Chávez Fragoso, 2001).

Debido a que los medios representan un recurso que permite la definición y comprensión de la realidad (McQuail, 2001), existen grupos, empresas e instituciones a través de los cuales se transmiten informaciones e ideologías determinados, con el propósito de influir la manera en la que la audiencia piensa sobre dichos temas (Gardner, Geierstanger \& Brindis, 2010; Van Hout \& Geert, 2008). Autores como Goffman (1974) indican que las formas en las que se producen los mensajes informativos, resaltan ciertos aspectos y opacan otros. Así, todo aquello que se emite al público en materia de salud influye en sus percepciones, ideas y actitudes respecto al tema, como riesgoso, urgente o alarmante (González \& Hernández, 2013). Por ello se considera importante la investigación sobre el fenómeno del periodismo especializado en salud.

\section{El contexto: Estado de Puebla}

La ciudad de Puebla es la cuarta ciudad más grande de la república mexicana, después del Distrito Federal, Guadalajara y Monterrey; ocupa el quinto lugar en población a nivel nacional y a la vez es la cuarta área metropolitana más grande de México, de acuerdo al último censo realizado por el INEGI (2010). Por su parte, el Estado de Puebla está en séptimo lugar como destino migratorio. El municipio capital, Puebla, tiene de acuerdo al último conteo de población, 1539819 millones de habitantes. El municipio que ocupa el segundo lugar, Tehuacán, tiene 274906 habitantes, Teziutlán en la tercera posición, con una población total de 92246 personas. Lo que resalta la importancia demográfica, económica y política del Estado.

La práctica periodística en Puebla surgió en la Nueva España y los albores de la independencia de México a la luz de un tema eminentemente político, que hasta la fecha sigue siendo primordial en los diarios (Sánchez, 2009). Puebla fue de las primeras ciudades en contar con una imprenta después de la ciudad de México; asimismo, fue de los primeros lugares en producir periódicos fuera de la capital (del Palacio Montiel, 2004). En sus inicios, la prensa en Puebla no solo era productora de información, sino también generadora de opinión pública (González Macías, 
2011), además de diversas formas de legitimación del poder. Con base en lo anterior, los medios a lo largo y ancho del territorio nacional favorecían un periodismo alineado con diferentes ideologías políticas (González Macías, 2011).

En Puebla la radiodifusión inició hasta 1936; pero no fue sino hasta 1987 cuando cobró presencia en Puebla el Proyecto Nacional de la Asociación de Concesionarios Independientes de la Radio (ACIR), integrándose al Grupo ACIR Nacional que cubre a toda la República mexicana (Anda Gutiérrez, 2004). El 1 de Junio de 2007 desaparece Grupo ACIR Puebla, propiedad de la Familia Cañedo, iniciando un nuevo corporativo radiofónico llamado Cinco Radio, que se mantiene hasta nuestros días, y controla siete estaciones. Del total de estaciones, cuatro tienen su sede en Puebla, el resto distribuye su programación en San Martín Texmelucan, Atlixco y Tehuacán.

La radio en el Estado de Puebla es importante en la vida cotidiana de las personas dado que es uno de los principales medios de información. La pertinencia de estudiar este medio es un tema vigente, sobre todo por la forma en la que se ejerce el periodismo en salud. Además, porque como medio de comunicación juega un papel determinante en la consolidación de la ideología que transmiten las instituciones dominantes. El abordaje de los temas en salud a través de la radio y del ejercicio de periodistas y comunicadores tiene que ver con el tipo de lugar en el que nos encontremos.

\section{Metodología}

Varios estudios han demostrado que los métodos de corte cualitativo favorecen una mejor y profunda comprensión de los fenómenos sociales, que resultaría compleja con la aplicación de métodos cuantitativos (Arroyave \& Blanco, 2005). Para esta investigación se utilizaron dos grandes enfoques cualitativos en el estudio de la cultura periodística: la sociología de la producción de noticias y la agenda setting.

La sociología de la producción de noticias busca identificar cuáles son los procesos y prácticas sociales que se llevan a cabo para producir los mensajes informativos por parte de los periodistas y comunicadores en los contextos de sus propios medios noticiosos, el cual es un trabajo empírico (de León, 2004). En la agenda setting, según lo propuesto por McCombs y Shaw (citados por Corral, 2008) los medios de comunicación tienen una gran influencia sobre el público al determinar qué historias poseen interés informativo y cuánto espacio e importancia debe dárseles. Con base en estos enfoques se exploraron, entre enero y marzo de 2015, un total de 11 radiodifusoras con sede en las ciudades de Puebla, Tehuacán y Teziutlán, que se detallan en el cuadro 1. 
Cuadro 1. Estaciones de radio que incluyen contenidos de salud en sus programaciones

\begin{tabular}{|c|c|c|}
\hline Ciudad & Radiodif & Programa \\
\hline Puebla & $\begin{array}{l}\text { Puebla FM } \\
\text { Acir Puebla } \\
\text { Imagen Radio Puebla } \\
\text { Grupo Cinco Radio }\end{array}$ & $\begin{array}{l}\text { Por ti, Puebla } \\
\text { Así sucede } \\
\text { Crónicas de salud } \\
\text { Buenos días con López Díaz }\end{array}$ \\
\hline Tehuacán & $\begin{array}{l}\text { Tehuacán FM } \\
\text { La Mejor FM } \\
\text { Ella FM } \\
\text { Stereo Luz }\end{array}$ & $\begin{array}{l}\text { Tehuacán noticias } \\
\text { Pulso informativo } \\
\text { Buenos días Tehuacán } \\
\text { Noticias al momento }\end{array}$ \\
\hline Teziutlán & $\begin{array}{l}\text { Teziutlán FM } \\
\text { XHFJ Radio Teziutlán } \\
\\
\text { XHOL Radio Impacto }\end{array}$ & $\begin{array}{l}\text { Mente sana } \\
\text { Revista } 95.1 \text { Exprés } \\
\text { Fórmula } 680 \text { noticias } \\
\text { Central } 910\end{array}$ \\
\hline
\end{tabular}

Fuente: Elaboración propia.

El estudio se centró en las producciones radiofónicas que identifican su eje temático como médico-sanitario, se distingue entre programas informativos y misceláneos o de revista. Se integró un análisis cualitativo basado en dos técnicas de investigación aplicadas:

1. Observación participante: instrumento apropiado para analizar el fenómeno de las culturas de producción de los periodistas en su propio contexto. La observación participante estuvo centrada en dos aspectos: a) Periodistas y comunicadores y b) Actividades que se realizan. La observación consistió en acompañar a los sujetos de estudio en su labor cotidiana de producir noticias y programas informativos. Periodistas y comunicadores estuvieron conscientes de la presencia de quien sustenta este estudio, como parte de una investigación de campo.

Se llevaron a cabo 11 sesiones de observación participante -incluyendo las entrevistas - por cada radiodifusora, las cuales tuvieron una duración de, por lo menos, dos horas. La duración de cada sesión estuvo determinada por el tiempo en el que ejecutan sus actividades los involucrados en el proceso de producción de los programas. El método de la observación participante resultó útil para contrastar, en el terreno de las prácticas periodísticas habituales, la información recogida en las entrevistas en profundidad. Soler (2011) indica que la serie de datos que se recogen en cualquier entrevista son solo enunciados verbales y que pueden existir discordancias entre lo que se asegura hacer y lo que en realidad se hace. De ahí la relevancia de recurrir a la observación participante como herramienta para complementar la información obtenida en las entrevistas, cuya aplicación se detalla a continuación.

2. Entrevista a profundidad: se basa en una guía de asuntos o preguntas para precisar conceptos u obtener mayor información sobre los temas deseados. En la entrevista a profundidad el entrevistador, según el curso que siga la interacción, tiene libertad de agregar los "porqués" que complementen la información. La entrevista a profundidad permitió entrar en diálogo con los sujetos de estudio y analizar los contrastes en sus respuestas.

Se realizaron entrevistas individuales a profundidad a 20 periodistas y comunicadores de radio, todos de la fuente de salud, a quienes se ofreció anonimato para su participación. Se consideraron, al menos, tres periodis- 
tas por radiodifusora, uno de los cuales ocupara cargo a nivel gerencial o directivo, con injerencia en la toma de decisiones administrativas, editoriales y de producción. Las entrevistas se efectuaron en sus lugares de trabajo y fueron grabadas bajo su propio consentimiento. Se hace referencia a los entrevistados como periodistas, comunicadores o productores, según el caso. Cuando es necesario para la discusión se ha hecho la distinción entre la sala de redacción, las cabinas de locución, los noticieros y los programas especializados o de revista. Dado el anonimato ofrecido a los participantes las citas textuales de las entrevistas se identifican con un número según el orden con el que fueron entrevistados, así como con el cargo que ocupan. Todo lo anterior permitió identificar la complejidad del fenómeno de las culturas de producción informativa en temas de salud en radiodifusoras poblanas: resultando en hallazgos que describimos a continuación.

\section{Hallazgos}

\subsection{Las fuentes en salud: ¿quiénes son?}

Tras la observación participante realizada en las 11 radiodifusoras tomadas como objeto de estudio, se encontraron situaciones semejantes: el método más común para obtener información es la entrevista directa, por lo que la labor de acopio de información se facilita. Producir temas y/o noticias de salud, para periodistas y comunicadores poblanos, es aparentemente sencillo: es suficiente con seleccionar un determinado número de fuentes informativas y entrevistarlas. Como afirma uno de los entrevistados: "Nosotros los periodistas simplemente tomamos la grabadora y nos vamos a nuestra primera fuente de información que, en este caso, podría ser algún servidor público, alguna dependencia estatal" (Periodista-productor, 4).

La cobertura recurrente a determinados agentes de información favorece que la propia fuente sea noticia por sí misma, evitando la cobertura de otros asuntos de salud pública en sus regiones. La relevancia de las fuentes se apareja a que se difunda lo que ella dice (Elías, 2008), sugiere o considera de interés: "Si el Director de Salud da una noticia es porque tiene que ser algo importante para la gente" (Editor, 11), "Si un médico tiene una campaña de diagnóstico o una institución la promueve, eso es de interés social para que cuiden su salud” (Reportera, 1), reflexionan los entrevistados. Al final, las fuentes son cada vez menos y más institucionalizadas pero con más poder, es decir, con mayor acceso al discurso en la radio. ¿Cuáles son esas fuentes? Hacemos una breve disertación sobre ello.

López (1995) sostiene que una fuente informativa será todo aquel canal - persona o institución - que proporciona datos sustanciales o complementarios para elaborar noticias. Martini (2000) apunta que las fuentes son los actores que el periodista entrevista, que suministran información. Lo que significa que la noticia es una construcción social elaborada a través de prácticas que tienen que ver con procesos de socialización y valoración de las formas de interpretar y presentar la realidad, ponderando relaciones sociales, políticas y hasta económicas por parte de los periodistas. Las siguientes opiniones describen cómo los periodistas validan la trascendencia de las fuentes institucionales: "Nosotros salimos, buscamos al funcionario, lo entrevistamos o en dado caso buscamos la opinión de la gente o ambas y contrastar" (Directora de noticias, 3). 
Primero voy a la fuente donde sé que me van a dar la información referente a las notas. También mi fuente es el ayuntamiento municipal para ver los programas sociales para el municipio. Si la persona está disponible le hago la entrevista, si es un funcionario con muchas ocupaciones hago una cita con esa persona (Reportera, 20).

Presentado el argumento, las rutinas periodísticas son pautas de actividades consensuadas por los periodistas como formas "válidas" y normales para producir contenidos. Los teóricos de la sociología de la producción de noticias han demostrado que los periodistas tienen un alto grado de rutinización y que sus métodos de recopilación, selección y presentación de noticias están estandarizados (Tuchman, 1991). De ahí el uso del término 'rutinas de producción' para referirse a los componentes que definen el trabajo diario de periodistas y comunicadores (funciones, normas, horarios, rondas y hasta presiones) que ellos mismos describen:

Nosotros los reporteros nos encargamos de la misma redacción. Cuando esa redacción se hace llegar a los jefes de información ellos son los que te dicen: "esta nota está mal, esta nota lleva muchos datos, puede ser muy aburrida para la lectura o para escucharla, acórtala” (Periodista, 5).

Primero que nada, la información la compartimos con el productor que es el que nos dirige y si lo ve necesario saca los temas a relucir, siempre y cuando en base a (sic) un especialista y ciertas preguntas que sean acorde[s] con el tema (Conductor, programa de revista, 16).

Identificado el nivel de influencia que la jerarquía organizacional tiene sobre el trabajo de periodistas y comunicadores poblanos, Altmeppen (2008) concibe a las salas de redacción como entidades que permiten a periodistas y comunicadores producir un canal informativo, por ejemplo un programa de radio. Por lo que la cobertura de los hechos traducidos en noticias no resulta del trabajo individual, sino que "los periodistas están incorporados en patrones organizacionales, los cuales, como estructuras preestablecidas, influyen en su trabajo y su comportamiento en cada sala de redacción" (p. 52) 3 . Para este autor el nivel organizacional resulta un elemento relevante en los estudios sobre periodismo porque permite identificar las condiciones y los contextos organizacionales en los cuales los contenidos informativos se producen.

Con lo antes expuesto, el marco del ejercicio periodístico es intersubjetivo, el periodista o comunicador está conectado con otros actores sociales en un marco de diferentes relaciones dentro y fuera del medio. En una de las observaciones participantes en la $95.1 \mathrm{Fm}$, durante un programa en vivo, el médico quiropráctico asistente le solicitó a uno de los conductores no ser entrevistado por su otra compañera, argumentando que el estilo que ella imprime en la forma de hacer entrevistas no le parecía adecuado, dado que lo consideraba aburrido y eso podría influir en que los radioescuchas no se interesaran en la información que él compartiría. El comunicador aceptó sin reparo la propuesta del entrevistado, quien después de haber concluido su "entrevista" se supo tenía contratados tres minutos de espacio al aire en ese programa para publicitar sus terapias de rehabilitación. Más que entrevista, resultó un mero monólogo en donde el comunicador no tuvo mayor intervención que la de ceder el uso de la palabra y afirmar lo que el quiropráctico exponía y, como cierre de "entrevista”, le preguntó la dirección de su consultorio, horario de atención y costos de consulta, enfatizando la importancia de que los radioescuchas asistieran a hacer uso de los servicios profesionales del médico. Se afirmó, por parte de los entrevistados, que se accedió a las peticiones del "invitado" por el hecho de haber pagado previamente el contrato de publicidad adquirido con la empresa, por lo que no podían negarse, pues eso habría ocasionado problemas con la gerencia general.

3 Traducción propia. 
Se puede afirmar que los contratos por publicidad acentúan el rol de mando que en la producción y divulgación de los temas en salud están asumiendo los personajes públicos que se consideran fuentes informativas especializadas y el sometimiento de periodistas y comunicadores a esas indicaciones bipartitas (empresa-cliente). Lo que sucede es una interacción que no está exenta de tensiones y conflictos, pero también de crecientes dependencias mutuas. La tendencia es informar acontecimientos de carácter institucional, sobre métodos diagnósticos y terapéuticos que satisfagan las necesidades económicas del médico, farmacéutica o empresa del ramo. Las fuentes de información periodística, identificadas para fines del presente estudio, son:

1. Funcionarios, directores y jefes de instituciones de servicio público relacionados a la salud (estatal, municipal o regional).

2. Empresas que prestan servicios profesionales de salud o medicina, con miras de contrato por publicidad.

3. Documentos, como boletines de prensa, páginas de Internet, que contienen datos que el periodista o comunicador considera útiles para satisfacer una demanda de información o conocimiento.

A partir del contacto con dichos agentes, la organización del trabajo de las salas de redacción se realiza en concordancia con los ritmos y pautas que devienen de las fuentes. El siguiente comentario lo confirma:

- ¿Cómo te acercas a las fuentes? - Primero por teléfono se trata, se hace una cita, cuando ellos llegan con mucho gusto te atienden, pero a veces por la agenda que ellos tienen te posponen la entrevista o te la cancelan. Entonces, en este caso de algún alto mando, alguna dependencia, alguna empresa, siempre se trata por teléfono y después estableciendo la cita se hace la entrevista. Con ellos simplemente es una entrevista formal. Hay algunos funcionarios que te van a decir: entrevístame de esto o entrevístame de otra situación. Claro, en el momento de la entrevista se retoman ese tipo de preguntas. Cosa que no puedes hacer con las áreas del ayuntamiento, puedes llegar y simplemente sacas tu grabadora y a quien te encuentres lo agarras para entrevista (Productor-periodista, 7).

Los reporteros sujetos de estudio se relacionan con sus fuentes informativas considerando los siguientes criterios:

a) Político-Institucional: Actores con determinada legitimidad suelen convertirse en fuentes informativas frente a otros actores sociales que no se presentan del todo en el medio (ong, pacientes, grupos civiles, etc.).

b) Comercial-Publicitario: Las relaciones comerciales que en los espacios informativos se mantienen también definen lo que es noticia o contenido de salud. A los anunciantes se les ofrece cobertura y difusión de ofertas especiales $\mathrm{o}$ actividades por el pago de la publicidad contratada.

Lo anterior no deja de interferir en los criterios de noticiabilidad (oportunidad, inmediatez, confiabilidad, verificación) que constituyen el eje a través de los cuales los periodistas interpretan la realidad y por ende la emiten a sus radioescuchas. En ese tenor, los entrevistados afirman: "Acuérdate que la noticia debe cumplir con cinco preguntas básicas, pero aparte que afecte a la sociedad, que afecte a toda una comunidad. Por ejemplo el desabasto (sic) de agua es noticia", (Editor - conductor, 16); "Somos un canal para que el funcionario vaya y hable, o dé a conocer un tema en particular" (Reportero-Co-conductor, 4). La jerarquización de esa noticiabilidad resulta compleja, situación que pesa en la autonomía de los mismos periodistas. La opinión de uno de ellos permite sostener el argumento:

Cualquier medio de comunicación que inicia con declaraciones fuertes con el paso del tiempo se suaviza, ¿por qué?, porque ya le llegaron al precio, porque tal funcionario, tal dependencia ya se anuncia ahí y ya 
tienes que ser hasta cierto punto tolerante con lo que comentes [...] El medio que te diga que es independiente está mintiendo, porque requieres de tus patrocinadores y desgraciadamente tocas intereses (Director de noticias - reportero, 7).

Es indiscutible que periodistas y comunicadores - siguiendo las indicaciones de sus editores, productores o directivos - asumen la información que proceda de estos organismos, limitando su contrastación y reproduciendo una visión parcial del asunto, que demuestra la falta de especialización en esa área. El hecho de que esas fuentes puedan tener un cierto interés en difundir aspectos de una determinada situación y dar publicidad a otros, y los mismos periodistas los reproduzcan sin mayores esfuerzos se traduce en que resulta más práctico cubrir una fuente oficial que realizar una cobertura propia con todo el costo, tiempo y trabajo que ello implica.

En el estudio de las culturas profesionales periodísticas Bonilla y Cadavid (2004) investigaron las funciones de los periodistas judiciales, encontrando que éstos parecen estar más preocupados por el rol de "transcriptores" de un suceso, que de mediadores que construyen sentido con su trabajo informativo, e identificaron la creciente dependencia de las fuentes institucionales y oficiales. La cultura de producción radiofónica aquí expuesta también evidencia el fenómeno de sometimiento a las fuentes informativas, como el caso de los boletines y comunicados de prensa, cuya influencia se describirá a continuación.

\subsection{Boletines de prensa: soluciones en el proceso de producción informativa}

Una situación que se identificó fue la recepción de boletines y comunicados de prensa. El uso que se hace de ese tipo de documentos por parte de los periodistas radica en que sirven para definir temas que pueden ser noticiables y verificarlos con sus funcionarios-fuentes. En aquellos boletines, que son enviados por las agencias de relaciones públicas, el periodista elimina la incertidumbre de a quién entrevistar, pues dichas empresas ofrecen y facilitan el acceso directo a médicos que tienen la encomienda de difundir productos o servicios de las empresas farmacéuticas o laboratorios que representan. Las respuestas son evidentes:

Sí, básicamente de laboratorios - ¿Qué haces con ellos?- Es el asunto de hablar de algún tema en específico, por ejemplo el asunto de la donación de sangre. Consiste en ofrecerte entrevistas con sus médicos como parte de su trabajo a cambio de que tú manejes algo trabajado a través de tal laboratorio o grupo farmacéutico. Es, si no constante, de vez en cuando ese tipo de invitaciones (Reportero, 2).

Sí, también mandan boletines de prensa a tu correo, tanto del gobierno municipal como del gobierno del Estado, o de algún funcionario en especial, algún diputado local, algún diputado federal que vaya a realizar, en coordinación con el sector salud, algún evento, entonces te van mandando boletines con anticipación (Reportera, 20).

A veces estar leyendo una nota texto es más tedioso que de pronto estar escuchando al funcionario que te está dando la información. Entonces, ya sabes que necesitas ir a ver al regidor o al director de salud para que te amplíe la información (Reportera, 1).

Nótese que las formas de producción de los contenidos de salud siguen caracterizándose por la pasividad del periodista, donde el circuito de investigación es reducido, con posibilidades de una visión sesgada. Sin embargo, los periodistas reconocen que los boletines de prensa no siempre son incluidos en sus informaciones, debido a la estructura y forma en la que se presentan, entendiendo que la radio, por la naturaleza de su lenguaje, exige formas 
atractivas para la emisión de contenidos. Empero, en la mayoría de las estaciones de radio investigadas el uso del lenguaje radiofónico, como recurso narrativo, no resulta adecuado, sobre todo porque la inmediatez con la que se produce la información limita la creatividad de los involucrados y resta dinamismo a los espacios radiales. Las citas explican algunos criterios por los que se valida la presentación de un comunicado de prensa:

A veces sí los pasamos, a veces no, es que no mandan audios. Se nos hace más complicado para nosotros leer, se hace complicado para la gente entender - ¿Por qué se hace complicado?-Como es de Puebla, viene con información, pues obvio, de la gran mayoría de hospitales de Puebla, no viene sectorizado por regiones, eso hace que la información no sea tan interesante para la gente de Tehuacán. Si tú les lees un boletín donde les estás informando del Hospital del Niño Poblano o de algún otro lugar no hay tanto impacto. Por ejemplo, ahora que fueron los festejos decembrinos, mandaron boletines sobre las grasas y ese rollo dando sugerencias, entonces ese boletín sí salió como un consejo para la gente (Directora de noticias, 3).

Lo que nosotros hacemos con esos boletines es archivarlos por prioridad. Claro, si es una información de algún servicio que la ciudadanía requiera, se menciona pero ya no como una nota de información, simplemente una mención dentro del noticiero: tal dependencia está haciendo esto, favor de pasar a sus oficinas que se encuentran en calle tal (Productor-reportero, 7).

Autores como Geert (2009) afirman que, quien escribe el boletín:

Busca satisfacer las expectativas del periodista [...] satisfacer las expectativas del periodista implica que el comunicado de prensa pueda ser copiado por los periodistas en sus propios reportes noticiosos [...] A través de la pre-formulación, los escritores de los comunicados de prensa proveen a los periodistas con fuentes escritas, las cuales han sido prefabricadas en un estilo noticioso apropiado y por lo tanto requiere de un mínimo de re-elaboración ( p. 506). ${ }^{4}$

Los boletines de prensa refuerzan los convenios publicitarios que se adquieran con empresas de relaciones públicas (Comunicación + Contenido y Edelman México son las que se identificaron en la investigación de campo) o farmacéuticas (Pfizer y MSD). Las relaciones comerciales que en los noticieros se mantienen determinan el contenido de salud: "Hemos tenido clientes que tienen que ver con la cuestión de la salud. Por ejemplo, una oftalmológica (sic), venía y explicaba en qué consistían sus tratamientos, cuáles son los métodos que ellos aplican" (Directora de noticias, 3). O como afirma otro de los entrevistados:

-Entonces ¿se da difusión de ese tipo de boletines? Sí se da difusión pero porque llegan a un acuerdo con la empresa en la parte comercial

- ¿Qué les ofrecen? La publicidad de la empresa, ellos pagan por difundirse y es parte también el hecho de que uno pueda tomar la entrevista con el médico o el químico que la empresa representa.

- ¿Cómo trabajas los boletines? Bueno, si son de las farmacéuticas o de los laboratorios esos se pasan directamente a la parte comercial y ellos ven si se emiten o no, y yo tomo en su momento cuando se hacen las notas o alguna entrevista con ellos pero de manera directa y ya no a través del boletín si no en contacto directo con el médico (Periodista, 9).

4 Traducción propia. 
Se puede decir que esta situación representa otra conceptualización pasiva acerca de la tarea cotidiana del periodismo en radiodifusoras regionales. Prescindir de aspectos en la información que contiene un comunicado de prensa y reproducir esa visión estrecha, en una nota informativa o un comentario significa tomar una serie de decisiones. Quien ha optado por hacer a un lado ciertos aspectos considera que las personas no pueden conocerlos debido a que no tienen la capacidad o criterios para analizar los contenidos presentados; otro de los móviles que llevan a reproducir textualmente la información es el interés de favorecer a la fuente que emite el comunicado o garantizar al periodista y comunicador el fácil acceso a la información sin necesidad de salir a buscarla:

Me parece interesante porque lo que hacen es acercarte un poco más al tema que ellos están manejando.

Lo que sí considero es que no tendría que ser nada más de esta manera y que ahí quede o que sea nada más durante las campañas que realizan, sino que deberían hacerlo de manera permanente para que así, por una parte, como reportero uno tiene más fuentes de información y segundo como empresa farmacéutica no sólo tendría mayor difusión sino que tendrían al alcance la manera de hacer llegar a la ciudadanía la información que están tratando de difundir (Periodista, 8).

Una especial crítica merece que el periodista no salga a buscar los "recursos" de su producto (noticia, programa, sección, cápsula informativa) sino que espere a que le lleguen, sin saber si existe otro mejor para elaborar sus contenidos informativos. Cualquier periodista debe recolectar, buscar, indagar sus insumos, siendo responsable de darle la forma adecuada en beneficio social.

Con estas consideraciones se puede afirmar que existe un proceso de producción periodística que va construyendo un sistema de relaciones bidireccionales a partir del interés de la fuente, cuya relación con el periodista y con el medio está marcada por el mutuo beneficio (compra-venta de publicidad, alcance de audiencias o consumidores). Nelkin (1990) sugiere que una forma de contrarrestar esta sumisión de la información es precisamente la especialización periodística, lo cual supone todo un reto.

Contar historias es un eje central del periodismo, pero con formas fáciles como la que resulta del acceso a los boletines de prensa (oficiales y empresariales), los géneros periodísticos (reportaje, crónica, artículo de fondo/ opinión) quedan relegados y subutilizados en el proceso de producción informativa dentro de las radiodifusoras. Se abordan los temas en salud a partir de las declaraciones de las fuentes impuestas por la jerarquía organizacional e institucional. Sin embargo, dichos documentos pueden resultar nichos de oportunidad en el sentido de que en ellos se identifican temas de interés periodístico, social y cultural, que devienen en una investigación a profundidad.

Con estas características del periodismo en salud en el Estado de Puebla, las audiencias son las que también resultan afectadas, pues el uso de información proveniente de determinadas fuentes favorece el sesgo en el periodismo, fomentando un fenómeno de contenidos dependientes.

\subsection{Cabinas de locución y salas de redacción: cultura de convergencia}

En lo que se refiere a las sesiones de observación participante en los espacios, cabinas de locución y salas de redacción, estas permitieron identificar cómo las rutinas de producción presentan aspectos comunes: trabajo de escritorio de los periodistas para elaborar sus notas informativas, acompañadas siempre de un audio o Insert -como ellos le llaman - para sustentar con declaraciones de los entrevistados sus noticias. En su mayoría son los 
mismos periodistas quienes graban y editan los audios que presentarán en los noticieros, como lo explica una de las periodistas entrevistadas:

Descargo los Inserts, un minuto como máximo, como es radio el tiempo es corto y después llega la hora del noticiero, entro a cabina para iniciar con mis otros compañeros periodistas el noticiero. Yo estoy en vivo dando la información de la nota que previamente armé escrita. Inicio, doy una introducción de lo que es la nota, después pongo un Insert que me lo mandan desde controles porque ya lo grabé previamente, donde ya el funcionario respalda todo lo que yo dije (Reportera, 20).

El quehacer cotidiano dentro de las estaciones de radio implica la ejecución de tareas simultáneas, pero permeadas por la pasividad, sobre todo por la influencia que tiene en su trabajo el uso de Internet:

Yo no acostumbro salir a reportear porque escucho, leo y comprendo del noticiero de televisión en el que también colaboro y de las redes sociales, asimilo lo que pasa diariamente aquí en la región y trato de dar un punto de vista y la opinión (Co-conductor, 18).

Con las rutinas de producción hasta este momento descritas, los noticieros de radio se organizan en emisiones de una hora que incluyen presentación de noticias en cadena, es decir, una seguida de la otra; secciones que van desde deportes, análisis político o cualquier otra, siendo la de salud, la de menos periodicidad:

Lo que pasa es que los noticieros tratan más los temas políticos o sociales. Tienen una importancia media. No podemos tener todos los días notas de salud porque aburres a la ciudadanía, a lo mejor le interesa lo que son los programas sociales, a lo mejor quiere saber de obras en pavimentación, en drenajes, a lo mejor quiere saber de educación (Periodista, 5).

Es interesante darse cuenta de que, a pesar de dedicarse a la divulgación de informaciones relacionadas con salud, los entrevistados reflejan que los mismos contenidos que recopilan, procesan y reproducen pueden ser desgastantes para la audiencia; de ahí que opten por incluir otros temas que puedan garantizar que los radioescuchas sigan sintonizando sus programas. Lo que se traduce en que los contenidos de salud queden velados y confundidos por asuntos que implican hablar de infraestructura, seguridad, obra pública, entre otros, mas no de salud o ciencias médicas:

Se les dedica el tiempo mínimo, la verdad. Porque son temas que no son tan interesantes para los coordinadores de noticieros, para los jefes de información - ¿Por qué?- Por el asunto de que no tienen, digamos, no sé si la fuerza, pero no son del agrado de ellos para abrir un noticiero, no están dentro de los segmentos; salvo que hubo una explosión en el hospital tal, entonces eso sí llama la atención, eres el primero en pasar (Periodista, 4).

En ese contexto, Morales y Vallejo (2011) constatan que los medios abordan cierto tipo de temas en correspondencia con unos indicadores como el impacto, la novedad y la afectación, que tienen que ver con la línea editorial, con los hábitos de los periodistas y también con las lógicas comerciales asociadas al rating. Chávez Fragoso (2001) subraya que existen escaso espacio y apoyo para la divulgación en salud por considerarlo poco noticiable; señala el error que a menudo se comete en los medios de comunicación en México al tratar información de ese tipo como una nota más, ponderando la novedad sobre el contexto y el entendimiento, haciendo un tratamiento más distante que un intento de hacer comprensible el conocimiento.

Para el caso de los programas de revista y los que se asumen especializados en salud, las rutinas de producción no dejan de verse afectadas por la pasividad en las formas de producción y presentación de contenidos. Los 
productores ejercen un papel importante, son ellos mismos los encargados de aprobar los contenidos en salud y quienes determinan quiénes serán los especialistas que formarán parte de las secciones que implican entrevistas en vivo:

Antes de entrar al aire el tema lo compartimos con el especialista, lo platicamos y de ahí sacamos las preguntas en base a (sic) lo que crea interesante [...] obviamente lo tenemos que dar a conocer a nuestro productor para que él nos dé el banderazo para poder emitir una información. (Conductores)

El programa es una vez a la semana, los martes. Tenemos que poner los temas de cada mes, de cada martes. (...) Yo soy simplemente el colaborador dentro del programa, soy como el moderador porque realmente la psicóloga es la que aborda los temas y soy el que toma las preguntas de mayor relevancia (Conductor, 6).

Como puede notarse, un elemento esencial en la producción de los contenidos es la entrevista, pues permite fundamentar la información que se divulga; más aún, permite solucionar la complejidad que refieren esos temas. Los comunicadores y presentadores tienen la tarea de moderar o presentar los contenidos, llevar el ritmo del programa de la manera más amena para el público: "Son temas que tratamos de tocar y hacer de forma muy amena para que la gente no se aburra escuchando el programa” (Productor, 13);

El tema de la salud es un tema complicadísimo, aparte de que debes manejar mucha terminología y debes de saber de qué estás hablando. Entonces, hemos sentido nosotros que debemos de ir como mezclando todo, tanto el humor, como la música, hacer más flexible el espacio (Conductor, 18).

La observación participante ayudó a constatar que las entrevistas que se realizan en las cabinas de locución, más que un género que demanda preguntas útiles para informar en profundidad y con sentido práctico a las audiencias, resultan en conversaciones donde no existe un orden claro de lo que se pregunta respecto al tema; evidencian una falta de especialización. A partir de ahí, los comunicadores se presentan como difusores de contenidos de diversa índole, lo que influye en cómo son vistos y abordados los temas de salud en las estaciones de radio poblanas.

Los temas de salud no son tan obligatorios dentro de la línea editorial que sigue el medio, casi nuestra línea editorial es más policiaca y la cuestión de la salud sí es importante pero no hay una línea marcada en donde se tengan que investigar ciertos temas, al menos de que sean de suma gravedad. Por ejemplo, la situación de algún brote de enfermedad. Entonces, a veces las notas de cuestión local tienen que ser más impactantes para poder pasar (Conductor de noticias, 3).

Sí se abordan, no son los principales porque el principal es la política. Es que también si tú te basas en algo en especial aburres a la ciudadanía, no podemos hablar siempre de salud, por lo regular variamos mucho (Reportera, 1 ).

Los comentarios dan cuenta de que las implicaciones del trabajo en las salas de redacción y cabinas de locución tienen que ver con la dependencia de agendas informativas para la producción de contenidos en salud. Sin embargo, el gran reto de la divulgación y el periodismo, y por ende de quienes trabajan y se interesan en ello, es el hecho de considerar dentro de su oficio una especialización, la cual es vista por los entrevistados como un crecimiento profesional necesario; pero es a su vez una labor que merece tiempo, inversión, vocación e interés: 
Una especialidad es un obstáculo muy importante porque tienes que estar especializado, para saber qué decir, para que también tu compañero sienta que tú también sabes y que pueda tener totalmente la confianza para que tú seas su apoyo (Productor, 10).

A veces el gran temor del periodista al hablar de ciertos temas es la situación del desconocimiento, a lo mejor sabemos lo básico - ¿Qué sería lo básico?- Pues, la situación de temas de enfermedades respiratorias que todo mundo sabe y de la situación de qué hacer, no automedicarse, etc. Pero ya temas más complejos pues no hay como el conocimiento pleno para estar informando ese tipo de temas (Directora de noticias, 3).

Diferentes investigaciones han demostrado que el periodismo padece muchas disfunciones. Guerrero y Márquez (2014) subrayan que el trabajo de las radios comunitarias en países latinoamericanos ha sido "capturado" no solo por actores económicos y políticos, sino por los discursos dominantes sobre lo que debe ser el periodismo. Como lo muestra Araya (en Guerrero \& Márquez, 2014) el trabajo de campo de las radios comunitarias en Chile continúa reproduciendo modelos verticales de comunicación en donde lo "alternativo" no está supeditado a la forma de conectarse con la comunidad ni en los procesos de socialización de la misma. Hay un emisor que articula y unas audiencias pasivas que pudieran "reaccionar". Uno de los cometidos principales del periodismo especializado en salud es facilitar la comunicación entre el mundo de la ciencia y la sociedad, y en el contexto de Puebla supone un reto.

La limitación informativa que se manifiesta, resultado de la metodología aplicada, puede generar expectativas que contribuyen, según Orueta et al. (2011) a la medicalización de la vida moderna, entendida como el proceso de convertir situaciones consideradas normales en cuadros patológicos que pretenden resolver -mediante la medicina- situaciones que no son médicas, sino sociales o de las relaciones interpersonales, es decir, la masificación de las consultas del médico familiar y de los servicios de urgencias.

Para seguir evidenciando los puntos que se deben reforzar en la cobertura de temas en salud es necesario investigar sobre la noción de profesionalismo, apoyada en una serie de ideales como la objetividad, la autonomía y la ética, que manifiesta o no el periodista en su labor y someterlos a un análisis minucioso para encontrar más alternativas de solución.

\section{Conclusiones}

Ante la complejidad del fenómeno de la producción informativa en radiodifusoras poblanas, el análisis permite verificar la hipótesis del origen de esta investigación:

1. La dependencia a las fuentes institucionales públicas y representantes del sector médico como parte de las rutinas que conforman el eje de una cultura profesional a nivel regional, una forma de sentido común dentro del ejercicio periodístico y un esquema estratégico. Las rutinas de producción identificadas hacen visible que la inmediatez con que se procesa la información impide fomentar la investigación y el contraste.

2. La rutinización de periodistas y comunicadores en la producción de las informaciones que difunden manifiesta incertidumbre en ellos mismos respecto de lo que implica no saber del todo qué es exactamente noticia de salud; lo cual resulta otro tema de investigación: ¿cuáles son las percepciones profesionales sobre cómo se concibe la salud en los medios? El periodismo hecho en nombre de la salud, desde Puebla, no ha alcanzado tal 
estatus en un sentido pleno. Los involucrados en la divulgación de contenidos de salud deben procurar el acceso a la mejor información disponible, rigiéndose en documentos que arrojen evidencias científicas de peso. Más aún, deben asumir que en ellos mismos está la base del cambio en el ejercicio periodístico.

3. La función de la política organizacional en la producción de contenidos en salud confirma que las radiodifusoras poblanas, antes que empresas de servicio público, son parte de conglomerados políticos y económicos. Los costos de producción -exigidos por la empresa- de los espacios informativos en radio, para la existencia y vigencia de los programas dentro de la programación diaria, son mitigados por la contratación de espacios publicitarios, pago por entrevistas y convenios entre el medio, los médicos, la empresa o institución que lo requiera. Lo que no deja de influir en el abordaje, encuadre y emisión de la información, por lo que detrás de los medios está el poder económico.

4. Es necesario invertir en el fortalecimiento de la capacidad de quienes participan en el quehacer informativo, proporcionando apoyo infraestructural, académico y de actualización que permita favorecer sus prácticas comunicativas y periodísticas. Se debe comprender la importancia del lenguaje propio de la radio; propiciar la creatividad, apoyada en todo momento en las herramientas narrativas como elementos sustanciales del periodismo. La experimentación de formas de estructurar y contar hechos son también puntos claves en los que se deben trabajar.

5. El aporte de esta investigación contribuye a identificar una serie de inquietudes y frustraciones que quienes ejercen el periodismo experimentan en su trabajo cotidiano, derivado de los niveles de influencia organizacional y económica. Estudios de esta naturaleza muestran una percepción cercana y real de una profesión que goza de popularidad, pero resulta una profesión de la cual no siempre se conocen muchos aspectos de su naturaleza desde dentro.

6. La mayoría de los programas radiofónicos investigados siguen modelos de tipo asimétrico, como el modelo del déficit (Lewestein, 2003) que entiende la comunicación como un proceso de recorrido unidireccional (que va del médico hacia el público) e ignoran las diferencias culturales que caracterizan a los actores involucrados en ese proceso (pacientes, comunidades originarias o rurales, entre otros). Esta investigación confirma la necesidad de reavivar el debate sobre los fines y los medios del periodismo especializado en salud. La ausencia de este debate en el contexto mediático regional supone el riesgo de ver impuesto el modelo de comunicación -podríamos atrevernos a decir, modelo de periodismo- más conocido, el del déficit. Lo ideal sería adoptar un modelo de comunicación interdisciplinario, que considere el aporte del enfoque diverso y sociocultural. Se propone que, desde dicho enfoque, se impulse la participación ciudadana, evitando quedarse solo en lo macro (discursos institucionales y fuentes preponderantes). Asimismo, la radio debe tomar conciencia de que es "un actor social" más, que debe participar activamente en la región de la que forme parte y hacer uso de su derecho en la construcción de una sociedad sana.

\section{Referencias}

1. Alcalay, R., \& Mendoza, C. (2000). Proyecto comsalud: Un estudio comparativo de mensajes relacionados con salud en los medios masivos latinoamericanos. Washington DC: Organización Panamericana de la Salud. Recuperado de http://www2.metodista.br/unesco/PCLA/revista7/projetos\%207-5.htm 
2. Altmeppen, Klaus D. (2008). The structure of news production: The organizational approach to journalism research. En: M. Löffelholz, y D. Weaver (orgs.), Global journalism research: Theories, methods, findings, future. (pp. 52-64) Nueva York/ Londres: Blackwell.

3. Anda Gutiérrez, C. (2004). Importancia de la radiodifusión en México. México: Noriega

4. Arroyave, J., \& Blanco, I. (2005). Cómo perciben los periodistas su profesión: entre el agotamiento y la fascinación. Investigación y desarrollo. 13(2), 364-389.

5. Bonilla, J. I., \& Cadavid, A. (2004). ¿Qué es noticia? Agendas, periodistas y ciudadanos. Bogotá: Pontificia Universidad Javeriana.

6. Chávez Fragoso, D. (2001). La divulgación de la ciencia en la radio de la ciudad de México. México: Dirección General de Divulgación de la Ciencia, unam.

7. Corral Velázquez, G. A. (2008). Análisis de las prácticas periodísticas en la Ciudad de Querétaro. (Tesis de Maestría en Comunicación, Universidad de Guadalajara). Recuperado de http://ccdoc.iteso.mx/cat. aspx?cmn=search\&fulltext=publisher\%3A\%22Era\%2F+Universidad+de+Guadalajara\%22\&pos=170

8. de León, S. (julio-diciembre, 2004). Prácticas periodísticas en Aguascalientes: estructuras de interpretación para acercarse al acontecer. Comunicación y Sociedad, (2), 185-228.

9. del Palacio Montiel, C. (julio-diciembre, 2004). La imprenta y el periodismo en las regiones de México (1539-1820). Comunicación y Sociedad, (2), pp. 161-184.

10. Elías, Carlos (2008). El periodismo científico como paradigma de la «noticia acatamiento». Una demostración desde las fuentes y una alerta de sus peligros. Revista Periodística, (11), 81-39. Doi: dx.doi. org/10.2436/20.3008.02.7

11. Gardner, A., Geierstanger, S., Brindis, C. (2010). Clinic consortia media advocacy capacity: Partnering with the media and increasing policy awareness. Journal of Health Communication, (15), 293-306.

12. Geert, Jacobs. (2009). Projected discourse: An analysis of receiver roles in press releases. Text, 4(18), 505-523. Doi: dx.doi.org/10.1515/text.1.1998.18.4.505

13. Goffman, E. (1974). Frame analysis, Harper and Row. Nueva York: Northeastern University Press.

14. González Macías, R. A. (2011). El periodismo mexicano: entre la modernidad y el atraso. Revista lberoamericana de Comunicación (21), 11- 40. Recuperado de http://revistas.ibero.mx/iberoamericana_de_comunicacion/ articulo_detalle.php?pageNum_paginas=0\&totalRows_paginas=30\&id_volumen=2\&id_articulo=13\&pagina=1\&pagina $=0$

15. González Santos, P., \& Hernández Domínguez, M. (2013). ¡Recuérdame! La obesidad infantil debatida y olvidada en los medios. Revista Iberoamericana de Comunicación (24), 45-69. Recuperado de http:// revistas.ibero.mx/iberoamericana_de_comunicacion/articulo_detalle.php?id_volumen=4\&id_articulo=46\&id_seccion=20\&active $=19 \&$ pagina $=2$

16. Guerrero, M. A., \& Márquez Ramírez, M. (2014). Media systems and communication policies in Latin America. United States: Palgrave Macmillan.

17. Hanitzsch, T. (2007). Deconstructing journalism culture: Toward a universal theory. Communication Theory, 17, 367-385.

18. Hernández Ramírez, M. E. (1997). La sociología de la producción de noticias. Hacia un nuevo campo de investigación en México. Comunicación y Sociedad, (30), 209-242. 
19. Hernández Sampieri, R., Fernández Collado, C., \& Baptista Lucio, P. (2003). Metodología de la investigación (3. ${ }^{\text {era }}$ edición). México: McGraw-Hill / Interamericana Editores S.A.

20. Herscovitz, H. G. (2004). Brazilian journalists' perceptions of media roles. Ethics and foreign influences on Brazilian journalism. Journalism Studies 5(1), 71-86.

21. Hornik, R. C. (1991). Alternative models of behavior change. En J. N. Wasserheit, S. O. Aral, \& K. K. Holmes (eds.), Research issues in human behavior and sexually transmitted diseases in the AIDS era. (pp. 201-217) Washington, DC: American Society for Microbiology.

22. Hughes, S. (2009). Redacciones en conflicto. El periodismo y la Democratización en México. México: Porrua.

23. Instituto Nacional de Estadística, Geografía e Informática. (2010). [Base de datos] México. Recuperado de http://www.censo2010.org.mx/

24. Lewenstein, B. (2003). Models of public communication of science and technology. [pdf] Recuperado de http://disciplinas.stoa.usp.br/pluginfile.php/43775/mod_resource/content/1/Texto/Lewenstein\%20 2003.pdf

25. Linden, T. (2003) Book Review: medical journalism: exposing fact, fiction, fraud. Public Understanding of Science, (12), p. 110.

26. López, M. (1995). Cómo se fabrican las noticias. Fuentes, selección y planificación. Barcelona: Paidós.

27. Martini, S. (2000). Periodismo, noticia y noticiabilidad. Buenos Aires: Norma.

28. McQuail, D. (2001). Introducción a la teoría de la comunicación de masas (3. era edición revisada y ampliada). Barcelona: Paidós.

29. Mercado Martínez, F.J., Robles-Silva, L., Moreno-Leal, N., \& Franco-Almazán, C. (2001) Inconsistent journalism: The coverage of chronic diseases in the Mexican press. Journal of Health Communication, (6), 235-247.

30. Montes de Oca, A. (2004). Periodismo en salud en revistas dominicales: ¿promoción o información? Apertura hacia el Futuro: Memorias de las V Jornadas Nacionales de Investigación Humanística y Educativa. Caracas: ucv/UCAB.

31. Morales, M., \& Vallejo, M. (julio-diciembre, 2011). Rutinas periodísticas y autopercepciones de los periodistas judiciales de los medios bogotanos. Signo y Pensamiento, 31, (59), 210-232.

32. Nelkin, D. (junio 8 de 1990). An uneasy relationship: The tension between medicine and the media, Lancet, 347(9015), 1600-16003

33. Orueta Sánchez, R., Santos Rodríguez, C., González Hidalgo, E., Fagundo Becerra E. M., Alejandre Lázaro, G., Carmona de la Morena, J. et al. (junio, 2011) Medicalización de la vida (I). Revista Clínica de Medicina Familiar, 4(2), 150-161. Recuperado de http://scielo.isciii.es/scielo.php?script=sci_arttext\&pid=S1699-695X2011000200011 \&lng=es

34. Sánchez Sánchez, S. (2009). Periodistas culturales en la ciudad de Puebla: sus prácticas, luchas y apuestas, Xochimilco: vam.

35. Salinas, C. (2008). El Plan Z: el periodismo no ha tenido lugar. En C. Lagos (comp.). El diario de Agustín (pp.245-250). Santiago de Chile: Lom.

36. Schudson, M. (2003). The sociology of news. Nueva York: Norton \& Company.

\section{3}


37. Soler, P. (2011). La investigación cualitativa, un enfoque integrador. En L. Vilches (coord.) La investigación en comunicación. Métodos y técnicas en la era digital (pp. 189-233). Barcelona: Gedisa.

38. Tuchman, G. (1978). Making news; a study in the construction of social reality. Nueva York: Free Press.

39. Tuchman, G. (1991). Qualitative methods in the study of news. En B. J. Klaus \& N. W. Jankowski (Eds). A. handbook of qualitative methodology for mass communications research (pp. 79-92). Londres: Routledge.

40. Van Hout T., \& Geert, J. (2008). News production theory and practice: fieldwork notes on power, interaction and agency. Pragmatics, 18(1), 59-85.

41. Waisbord, S. (2000). Watchdog journalism in South America. News, accountability and democracy. Nueva York: Columbia University Press. 


\section{ESTUDIOS}

\section{A NEXOS}

Correo electrónico que muestra la forma en la que se contacta al periodista

Además del gusto de saludarte, y como seguimiento a la entrevista prevista con el Dr. David Blumenkron, experto en esclerosis múltiple. El dactor me comenta que si es posible

que la entrevista se realice a las 13:00 hrs., quedo atenta a tus comentarios.

Maria Picazo

Adjunta encontrarás información sobre la enfermedad y la biografía del especialista.

Añadir a circulos

Estay a tus órdenes.

Saludos cordiales,

Maria

María Picazo I Comunicación + Contenido I Relaciones Públicas

Tatavasco No. 31. Col. Santa Catarina. Coyoacán.

Tels. 55546591 ext 13 Cel. 5539080300

maria.picazo@comunicacionmascontenido.com I maria.picazo.maldonado.coco@gmail.com

Co.Co

2 archivos adjuntos

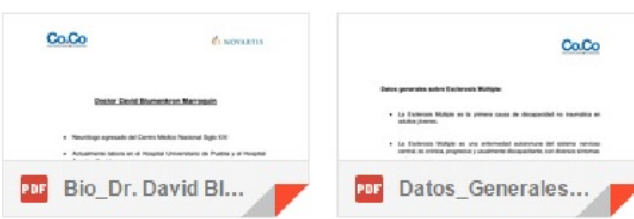

Compartido de común acuerdo por uno de los entrevistados 